\title{
Distribution of Pathogenic Yeasts in Different Clinical Samples: Their Identification, Antifungal Susceptibility Pattern, and Cell Invasion Assays
}

This article was published in the following Dove Press journal: Infection and Drug Resistance

\author{
Satish T Pote ${ }^{1,2}$ \\ Mahesh S Sonawane \\ Praveen Rahi ${ }^{\prime}{ }^{\prime}$ \\ Sunil R Shah ${ }^{3}$ \\ Yogesh S Shouche' \\ Milind S Patole' \\ Madhuri R Thakar ${ }^{2}$ \\ Rohit Sharma'
}

'National Centre for Microbial Resource (NCMR), National Centre for Cell Science, NCCS Complex, S.P. Pune University, Pune 4II 007, Maharashtra, India; ${ }^{2}$ National AIDS Research Institute, Pune 4I I026, Maharashtra, India; ${ }^{3}$ Bharati Vidyapeeth Deemed University Medical

College, Bharati Vidyapeeth, Pune

4II043, Maharashtra, India
Correspondence: Rohit Sharma National Centre for Microbial Resource (NCMR), National Centre for Cell Science, NCCS Complex, S.P. Pune University, Ganeshkhind, Pune 4II 007, Maharashtra, India

Tel +9l-20-25329035

$\mathrm{Fax}+91-20-25692259$

Email rohit@nccs.res.in
Introduction: Species of genus Candida are part of the common microbiota of humans; however, some of the Candida species are known opportunistic pathogens. Formation of biofilms, resistance to antifungal drugs, and increase in asymptomatic infections demands more studies on isolation, identification and characterization of Candida from clinical samples. Methods: The present manuscript deals with assessment of authentic yeast identification by three methods viz., DNA sequencing of $28 S$ rRNA gene, protein profiles using MALDI-TOF $\mathrm{MS}$, and colony coloration on chromogenic media. Antifungal susceptibility and in vitro cell invasion assays were performed to further characterize these isolates.

Results: Comparison of three methods showed that DNA sequence analysis correctly identified more than $99.4 \%$ of the isolates up to species level as compared to $89 \%$ by MALDI-TOF MS. In this study, we isolated a total of 176 yeasts from clinical samples and preliminary morphological characters indicated that these yeast isolates belong to the genus Candida. The species distribution of isolates was as follows: 75 isolates of Candida albicans (42.61\%), 50 of C. tropicalis (28.40\%), 22 of C. glabrata (12.5\%), 14 of C. parapsilosis (7.95\%) and 4 of Clavispora lusitaniae $(2.27 \%)$. Other species like Cyberlindnera fabianii, Issatchenkia orientalis, Kluyveromyces marxianus, Kodamaea ohmeri, Lodderomyces sp., and Trichosporon asahii were less than $2 \%$. Antifungal susceptibility assay performed with 157 isolates showed that most of the isolates were resistant to the four azoles viz., clotrimazole, fluconazole, itraconazole, and ketoconazole, and the frequency of resistance was more in non-albicans Candida isolates. The susceptibility to azole drugs ranged from $7 \%$ to $48 \%$, while $75 \%$ of the tested yeasts were susceptible to nystatin. Moreover, 88 isolates were also tested for their capacity to invade human cells using HeLa cells. In vitro invasion assay showed that most of the $C$. albicans isolates showed epithelial cell invasion as compared to isolates belonging to C. glabrata, C. parapsilosis and C. tropicalis.

Discussion: The identification of yeasts of clinical origin by sequencing of $28 S$ rRNA gene performed better than MALDI-TOF MS. The present study reiterates the world scenario wherein there is a shift from Candida strains to emerging opportunistic pathogens which were earlier regarded as environmental strains. The present study enlightens the current understanding of identification methods for clinical yeast isolates, increased antifungal drug resistance, epithelial cell invasion as a virulence factor, and diversity of yeasts in Indian clinical samples.

Keywords: yeast clinical isolates, chromogenic media, DNA sequencing, MALDI-TOF MS, antifungal drug resistance

\section{Introduction}

Opportunistic infections of cutaneous, mucosal, bloodstream or deep-seated organ termed as candidiasis caused by members of genus Candida have become a major 
threat to humans. Persons on long-term treatment for malignant or infectious diseases, and with reduced immunity are prone to candidiasis. ${ }^{1}$ Candida albicans accounts for a major fraction of morbidity and mortality, however, other Candida species including C. glabrata, C. parapsilosis, C. tropicalis and Issatchenkia orientalis (=C. krusei=Pichia kudriavzevii) were found to be responsible for invasive diseases. ${ }^{2}$ The non-albicans Candida causing infection show less sensitivity to the antifungal agents used in practice by dermatologists affecting the therapeutic rate of candidiasis. Increase in fatal cases of candidiasis is also attributed to failure to commence accurate anti-Candida therapy at an appropriate time. ${ }^{3}$ Therefore, it is most important to correctly identify the species of Candida and determine the choice of therapeutic agent for early management of disease.

Existing techniques for species identification of Candida may be time consuming and even result in nonauthentic identification if carried out by classical usage of blood culture techniques. Blood cultures are sensitive up to a single colony-forming unit per $\mathrm{mL}$ of blood, however the time taken to obtain positive results is too long, and may require as long as 8 days for a few samples. ${ }^{4}$ Detection of $\beta$-D-glucan, a cell wall constituent of Candida, carbohydrate assimilation test, and biochemical profiling can also rapidly identify Candida species. ${ }^{5,6}$ However, the above methods can only identify some species of Candida due to lack of available assays or resolving power for cryptic species. Many of the recently developed molecular methods are real-time PCR assays that provide rapid results. ${ }^{7-9}$ However, they are often limited in the number of species that can be detected in a single assay and difficulty to detect new pathogenic yeasts or strain variations. It is observed that even though real-time PCR was specific it does not improve the diagnostic sensitivity of Candidaemia and invasive Candidiasis. ${ }^{10}$ Standard PCR-based assays and its modifications are also limited in the number of species that can be identified from a single amplification and lack of species-specific markers for all pathogenic yeasts. ${ }^{11}$ Nonetheless DNA sequencing of amplified PCR products continues to be the most reliable method for authentic fungal (both mycelia and yeast forms) species identification. ${ }^{12}$ Relatively recently, Matrix-assisted laser desorption/ionization time-of-flight mass spectrometry (MALDI-TOF MS) has also been employed successfully for a rapid identification of five major species of the genus Candida. $^{13}$ Direct MALDI-TOF MS assay of blood culture broths is also effectively employed for identification of Candida species. ${ }^{14}$ Apart from the above methods, chromogenic agar as a differential culture medium is also commonly used by pathologists and has so far helped in isolation and rapid presumptive identification of clinically important Candida species. $^{15}$

Studies on clinical candidiasis in India have been neglected even though they are a major cause of mortality in immuno-compromised patients. ${ }^{16-19}$ In the present study, comparison of three different techniques was carried out for yeast species identification viz., PCR amplified partial ribosomal DNA gene sequence, MALDI-TOF MS and color developed on chromogenic agar for yeast pathogens identified initially by germ tube assay. Characterization of these clinical isolates for antifungal susceptibility and invasion capability were also performed. We also report the diversity of yeast species from different clinical samples collected from hospitals and epidemiology of candidiasis.

\section{Materials and Methods Sample Collection, Yeasts Isolation and Characterization}

Clinical samples were obtained from our outpatients department (OPD) and intensive care unit (ICU) patients of Bharati Hospitals, Pune, Maharashtra, India with prior approval of the Institutional Ethics Committee. The time period of sample collection was from May 122014 to May 12 2017. Maximum samples were obtained from urine (77), followed by sputum (33), vaginal swabs (25) and blood (15). Other clinical samples like pus, oral swab, cerebral spinal fluid (CSF), broncho-alveolar lavage and trans-bronchial biopsy, peritoneal and pericardial fluid, endotracheal tube (ETT), and cornea accounted for 26 samples. Initially, germ tube and hyphal formation were induced as per the standard protocol for which a solution containing tryptic soy broth and fetal bovine serum was employed. $^{20}$ Then they were microscopically examined to find the presence of any unicellular yeast cells, pseudohyphal or true hyphal bodies. Samples with probable yeast were plated on Saboraud Dextrose agar (SDA) with chloramphenicol and incubated at $30 \pm 0.1^{\circ} \mathrm{C}$ for 7 days. Well isolated colonies were further processed to obtain pure cultures which were deposited at the National Centre for Microbial Resources, Pune, India. Colony characteristics of pure cultures were noted. Biochemical characteristics for a selected few isolates belonging to $C$. albicans group 
were performed using API Candida strips (bioMérieux SA, France) with twelve identification tests for sugar acidification and enzymatic reactions.

\section{PCR Amplification, DNA Sequencing, and Phylogenetic Analysis}

Genomic DNA from each isolate was extracted using lithium acetate. Briefly, a single colony obtained from pure culture was mixed well in $200 \mu \mathrm{L}$ of lithium acetate $(0.2 \mathrm{M}$ in $1 \%$ sodium dodecyl sulphate) and incubated at $70 \pm 1^{\circ} \mathrm{C}$ for 1 hour. Subsequently DNA was precipitated using $300 \mu \mathrm{L}$ absolute ethanol. The precipitated DNA was solubilized in Tris:EDTA and used for PCR amplification of 28S rRNA gene (Large Subunit - LSU) using primers LROR (ACCCGCTGAACTTAAGC; at nucleotide position 24 42) and LR5 (TCCTGAGGGAAACTTCG at nucleotide position 964-948). To compare sequences of different species of Candida for species-specific nucleotide changes which can act as species-specific markers, multiple alignment was done using $28 \mathrm{~S}$ rRNA gene sequences. The ITS region was also amplified using ITS1 (TCCGTAGG TGAACCTGCGG at nucleotide position 1769-1787 in 18S rRNA gene) and ITS4 (TCCTCCGCTTATTGAT ATGC at nucleotide position $41-60$ in $28 \mathrm{~S}$ rRNA gene) primers. The amplification was confirmed on $1.0 \%$ agarose gel after ethidium bromide staining. PCR product was purified by $\mathrm{PEG}-\mathrm{NaCl}$ and was sequenced using the BigDye Terminator Cycle Sequencing Ready Reaction kit and ABI 3730xl automated DNA sequencer having Sequence Scanner software for the sequence quality analysis (Applied Biosystems,

Thermo Fisher Scientific, Waltham, MA, USA). Sequences generated were manually edited using ChromasPro version 1.34 and saved in FASTA format. The isolates were identified up to species level using NCBI BLASTn search tool with type and non-type sequences. ${ }^{21}$ The results of the BLASTn search are compiled in (Table S1) with their NCBI accession number.

A phylogenetic tree was constructed individually for each strain to confirm its species identification using known sequences of different Candida spp. available in GenBank at NCBI. It is done because in many cases it is observed that two or more hits in BLASTn results are with closest similarity of $99 \%$. In such cases, it becomes difficult to assign the species name to the unidentified isolate and a phylogenetic tree construction with authentic sequences from NCBI GenBank helps in correct identification. As the
BLASTn results are not from a rigorous method and just based on pairwise comparison and scores, the phylogenetic analysis is more robust which uses multiple sequence alignment giving correct evolutionary relationship. The combined phylogenetic relationships of the isolates with other species of yeasts were examined using 28S rRNA gene sequences. Sequences corresponding to type strains were retrieved from NCBI including an out-group, Saccharomyces cerevisiae (NR111007). Multiple alignments of all sequences were carried out using ClustalW program in MEGA $7 .^{21}$ The phylogenetic tree was generated by bootstrap test Neighbour joining (NJ) with Kimura twoparameter model. The stability of internal nodes was assessed by bootstrap analysis with 1000 replicates.

\section{Identification of Isolates Using MALDI-TOF/ MS Biotyper and Chromogenic Media}

Actively growing pure cultures were used for protein extraction for MALDI-TOF MS analysis. Briefly, a single colony of each isolate was inactivated in $75 \%$ ethanol, and then suspended in a $70 \%$ formic acid and subsequently an equal volume of acetonitrile was added. The resulting extract was directly layered over 384-spot polished MALDI steel target plate. A saturated solution of MALDI matrix (HCCA, Bruker Daltonik) was applied to each sample and dried. Measurements were performed with Bruker Biotyper Autoflex using "Flex Control" (Compass Flex Series version 1.3) software and a $60 \mathrm{~Hz}$ nitrogen laser (337 nm wavelength). Spectra were collected in the linear positive mode in a mass range covering $1960-20,132 \mathrm{~m} / \mathrm{z}$. Spectra ranging from the mass-to-charge ratio (m/z) 2000-20,000 were analyzed using MALDI Biotyper automation control and the Bruker Biotyper 3.1 software and library. Identification score value between 2.000-3.000 indicated species-level identification, scores of 1.700-1.999 indicated genus-level identification, and scores of 1.700 or less indicated no identification. For screening yeasts on chromogenic media, isolates were streaked using four quadrant methods on the solid HiCrome ${ }^{\mathrm{TM}}$ Candida Differential Agar (HiMedia Laboratories, India) and incubated at $37 \pm 0.1^{\circ} \mathrm{C}$ for 48 hours. The color of the colony was noted and identification was made using colony color and appearance as described by the manufacturer.

\section{In Vitro Cell Invasion Assay}

In vitro invasion assay was done using cover slip cultures of HeLa cells $(1,00,000$ cells per cover glass) maintained on minimal essential medium with Earle's salts and 10\% 
foetal bovine serum. A single yeast colony was inoculated in $1 \mathrm{~mL}$ of complete medium and incubated for 3 hours at $37 \pm 0.1^{\circ} \mathrm{C}$ in $5 \%$ carbon dioxide atmosphere. After the germination of ovoid yeast cells to hyphae, cell suspension was diluted in 1:4 with complete medium and coincubated with the HeLa cells (multiplicity of infection of 10). Cells were incubated for 3 hours at $37 \pm 0.1^{\circ} \mathrm{C}, 5 \%$ $\mathrm{CO}_{2}$. After co-incubation, the cell sheet was rinsed twice with growth medium and cells were fixed with $3.7 \%$ paraformaldehyde (PFA) for 10 minutes. Further, cells were permeabilized with $0.05 \%$ Triton X-100 for 5 minutes, followed by thorough washing with phosphatebuffered saline. After permeabilization, cells were stained using FITC-Phalloidin $(10 \mu \mathrm{g} / \mathrm{mL}$; Sigma Aldrich) and DAPI $(0.04 \mu \mathrm{g} / \mathrm{mL}$; Sigma Aldrich) by incubating in the dark for 30 minutes. Then cells were rinsed with PBS and cover slips were mounted on glass slides using mounting media. The confocal images were acquired using Zeiss LSM 510 meta Confocal Laser scanning microscope.

\section{Antifungal Susceptibility Test}

Antifungal susceptibility testing was conducted according to the manufacturer's instructions which comply with Clinical Laboratory Standards Institute (CLSI) guidelines. The antifungal agents included in the susceptibility testing plates included clotrimazole $(10 \mu \mathrm{g})$, fluconazole $(25 \mu \mathrm{g})$, itraconazole $(10 \mu \mathrm{g})$, ketoconazole $(10 \mu \mathrm{g})$ and nystatin (100 unit) (HiMedia Laboratories, India). For antifungal susceptibility analysis each yeast strain was plated by pour plate technique. Discs with antifungals were applied and the zone of inhibition was measured after the 24 hours of incubation.

\section{Results and Discussion Identification of Yeasts by Sequencing and MALDI-TOF MS}

A total of 176 isolates were analyzed by MALDI-TOF MS. The results of the DNA sequencing and MALDITOF MS for correct identification were compared which showed that the MALDI-TOF MS could identify 157 yeast isolates correctly. The proportions of accurate identifications of different species of Candida and other yeast strains by MALDI-TOF MS and sequencing are shown in Table 1. The correct identification rates of $C$. albicans and $C$. glabrata by MALDI-TOF MS were more than $90 \%$ whereas for $C$. parapsilosis and $C$. tropicalis they were more than $80 \%$. Overall, the correct identification rates of the 176 yeast isolates to species levels by the Bruker
MALDI Biotyper systems was $89.2 \%$. Chao et $\mathrm{al}^{22}$ observed $92.5 \%$ correct identification of 200 yeasts to either species or a species complex by MALDI-TOF MS. As shown in different reports, the accuracy of yeast identification by MALDI-TOF MS varies from 84 to $99 \%$. $^{23-29}$ In the present study, MALDI-TOF MS used several characteristic peaks for differentiating $C y$. fabianii, K. marxianus, Ko. ohmeri and T. asahii from other yeasts, thus correctly identifying them. Although this study is not the first to report using characteristic MALDI-TOF MS spectra to separate and successfully identify non-candida yeasts like Cy. fabianii, K. marxianus, Ko. ohmeri and T. asahii, it confirms that MALDI-TOF MS presents an effective alternative to sequencing for the correct identification of the emerging yeast pathogens (Figure S1). MALDI-TOF MS gives fast, authentic identification at a lower cost than PCR, DNA sequencing, or microbiological techniques such as phenotypic and biochemical characters commonly used in clinical laboratories. It is now possible to obtain high quality spectra for pathogen lysates and generate a spectral library by improving the mass spectrometer and software used for analysis. Moreover, improvements in the extraction procedures are also yielding sufficient amounts of proteins from limited amounts of clinical samples for spectral analysis. In general, there are three types of extraction procedures for obtaining protein lysates from microorganisms: the direct colony method (without treating with lysing agent), the extraction method (which involves on plate extraction, ethanol-formic extraction and trifluoracetic acid extraction), and the disruption method (which involves physical extraction and enzymatic lysis extraction). ${ }^{30,31}$ There is an urgent need to standardize the method of protein extraction for the newer environmental isolates which are now being reported as emerging pathogens. ${ }^{29}$ Thus, based on the above results, apart from DNA sequencing, MALDI-TOF MS presents an efficient alternative method for authentic identification of pathogenic yeasts, however, there is a need to improve the database by regular addition of newer yeast species from both clinical and environmental scenarios.

\section{Identification Efficiency of Chromogenic Media}

Chromogenic media is used commonly in clinical laboratories for rapid identification of Candida isolates. It can identify C. albicans, C. tropicalis, C. glabrata and I. orientalis based on the color response to the medium and colony morphology 
Table I Comparison of Species Identification of Clinically Obtained Yeast Species with MALDI-TOF MS and Hi Chrome chromogenic media

\begin{tabular}{|c|c|c|c|c|c|}
\hline \multirow[t]{2}{*}{ Species } & \multirow{2}{*}{$\begin{array}{l}\text { Identification By } 28 \mathrm{~S} \text { rRNA } \\
\text { Gene Sequencing }\end{array}$} & \multicolumn{2}{|c|}{ Correct Identification } & \multicolumn{2}{|c|}{ Misidentification } \\
\hline & & $\begin{array}{l}\text { Chromogenic } \\
\text { Media }\end{array}$ & $\begin{array}{l}\text { MALDI- } \\
\text { TOF MS }\end{array}$ & $\begin{array}{l}\text { Chromogenic } \\
\text { Media }^{a}\end{array}$ & $\begin{array}{l}\text { MALDI- } \\
\text { TOF MS }\end{array}$ \\
\hline Candida albicans & 75 & 64 & 71 & $7(4)$ & 4 \\
\hline Candida glabrata & 22 & 19 & 20 & $0(3)$ & 2 \\
\hline Candida tropicalis & 50 & 40 & 42 & $5(5)$ & 8 \\
\hline Issatchenkia orientalis & 2 & 0 & 2 & 0 & 1 \\
\hline Candida africana & I & 0 & 0 & $0(I)$ & 1 \\
\hline Candida parapsilosis & 14 & 0 & 12 & $13(I)$ & 2 \\
\hline Clavispora lusitaniae & 4 & 0 & 3 & 4 & 1 \\
\hline Cyberlindnera fabianii & 2 & 0 & 2 & 2 & 0 \\
\hline Kluyveromyces marxianus & 3 & 0 & 3 & 3 & 0 \\
\hline Kodamaea ohmeri & 1 & 0 & I & I & 0 \\
\hline Lodderomyces sp. & 1 & 0 & 0 & I & I \\
\hline Trichosporon asahii & I & 0 & 1 & I & 0 \\
\hline Total & 176 & 123 & 157 & $39(14)$ & 19 \\
\hline Percentage (\%) & 100 & 70 & 89 & $22(8)$ & I I \\
\hline
\end{tabular}

Note: ${ }^{\text {T}}$ The numbers inside parentheses are not identified by chromogenic media.

(Table 1, Figure S2). Out of 75 isolates identified as C. albicans by sequencing and 71 by MALDI-TOF MS, only 64 were identified as $C$. albicans by coloration using chromogenic media. Seven isolates were misidentified and four remained unidentified. These misidentified and unidentified isolates were correctly identified by sequencing as $C$. tropicalis and Cl. lusitaniae. Out of 22 and 20 isolates that were identified by sequencing and MALDI-TOF MS respectively as $C$. glabrata, 19 were identified by chromogenic media and three were not identified. Out of the 50 isolates of $C$. tropicalis, chromogenic media identified 40 of them and five were misidentified and five were not identified. Out of two isolates that chromogenic media identified $I$. orientalis, both were authenticated by DNA sequencing and MALDI-TOF MS. However, the misidentified isolates of $I$. orientalis by chromogenic agar were correctly identified by sequencing as Cy. fabianii, Meyerozyma caribbica and Wickerhamonyces anomalus. Moreover, the characteristic coloration of non-Candida yeasts such as $C y$. fabianii, K. marxianus, T. asahii, I. orientalis and Ko. ohmeri are not defined in the literature (Figure S3). With the increase in the different types of clinical isolates and changes in the taxonomy of yeast, it is now essential that we do not rely on chromogenic agar techniques for yeast identification.

\section{Antifungal Susceptibility Test}

Invasive fungal infections are becoming common and their rapidity of invasiveness by pathogen demands early arrest of infection by antifungal agents. There are immense changes in the host factors, infecting fungi, and antifungal agents and hence there is a need to accomplish antifungal susceptibility tests. A total of 157 isolates were tested for their susceptibility to antifungal drugs and out of that, 104 isolates were resistant to itraconazole, 98 were resistant to fluconazole, 82 were resistant to clotrimazole and 70 were resistant to ketoconazole (Figure 1, Tables S2 and S3, Figure S3). The increased resistance of clinical yeasts to fluconazole $(55 \%)$ is of concern as it is one of the most common antifungals used for treatment of candidiasis. Strains of C. glabrata showed high resistance to all the antifungals tested followed by $C$. tropicalis. Overall, strains of C. albicans and $C$. parapsilosis were susceptible to the antifungals tested. Some of the emerging pathogens like T. asahii, Ko. ohmeri and $\mathrm{Cl}$. lusitaniae were found to be sensitive to most of the tested antifungal agents. However, isolates of $I$. orientalis were resistant to all the tested antifungal agents. Thus, drug resistance data of yeasts other than Candida will help in the proper treatment of such infections. These resistance rates are higher as compared to earlier published studies. $^{32-37}$ Earlier studies have reported that high concentrations of ketoconazole and fluconazole reduce the adhesion of $C$. albicans to buccal epithelial or endothelial cells suggesting an azole-host cell specific effect. ${ }^{38}$ Fluconazole and voriconazole have also been shown to reduce tissue destruction in reconstituted esophageal epithelia after $C$. albicans infection. ${ }^{37}$ Martins et $\mathrm{al}^{39}$ found that the cure rates of fluconazole for vaginitis caused by $C$. albicans 


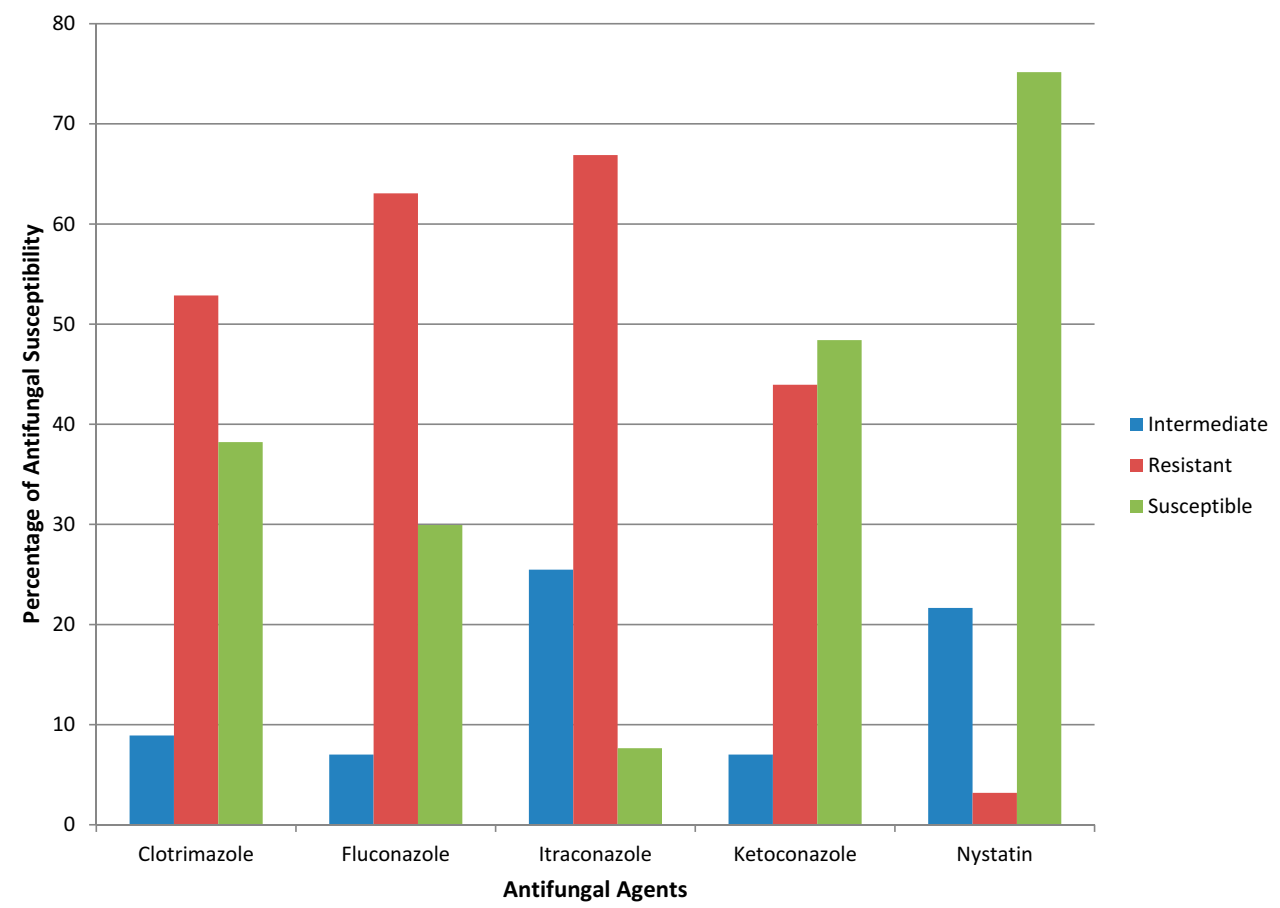

Figure I Percentage of in vitro antifungal susceptibility testing of five antifungal agents (i.e., clotrimazole, fluconazole, itraconazole, ketoconazole, nystatin) against 157 yeasts isolated during the study.

was $82 \%$ and for non-albicans species was $100 \%$. However, the cure rates for vaginitis due to $C$. albicans treated with nystatin was $93 \%$, which severly dropped further to $44.4 \%$ when non-albicans species-infected patients were treated with nystatin. However, in our study, nystatin was found to be a better antifungal agent as compared to azoles. In the present study we observed that $80 \%$ of the strains of C. glabrata were resistant to the azole antifungal agents. It has been observed that strains of $C$. glabrata have highest resistance among various species of Candida. ${ }^{40,41}$ Many researchers have also isolated multi-drug resistant strains of C. glabrata. $^{42,43}$

\section{In Vitro Cell Invasion Assay}

The invasion of host cells by the pathogenic yeast cells is one of the important virulence factors in invasive candidiasis. Most of the studies on epithelial cell invasion are carried out using C. albicans. ${ }^{38,44}$ Yeast adhesion to the epithelium is essential for colonization and invasion and the passive processes of hydrophobic and electrostatic attraction mediates this interaction. In this study, we investigated the ability of various yeast isolates to invade epithelial cells. A total of 88 yeast strains were tested for in vitro cell invasion assay using HeLa cell line. Actin remodeling of the host cell line around the invading hyphae as seen by staining with fluorescent dye FITC-phalloidin was considered as an invasion event. Although all 88 yeast isolates tested were isolated from invasive candidiasis patients, only a few could show invasion in vitro. Out of 58 isolates of C. albicans tested for invasion only $86 \%$ showed cell invasion in vitro. A representative image of one of the isolates of C. albicans (isolate no. 8_1) clearly showed that it has invaded the epithelial cell line (Figure 2). However, none of the eight isolates of C. glabrata tested showed any invasion. While only a single isolate of C. parapsilosis (out of 9 isolates tested) and C. tropicalis (out of 13 isolates tested) showed HeLa cell invasion (Figure S4 and S5). It has been observed that yeast cells invade the epithelial cells with the help of pseudo-hyphae or hyphal structures. Sometimes yeast forms can also be efficiently internalized by epithelial cells, as seen in the case of $C$. parapsilosis in this study (Figure S5). Previously, it was observed that the yeasts infect with their hyphal stage and convert to yeast form once inside the endothelial cells. The hyphal stage helps in providing more strength and is enzymatically and metabolically more active, helping in establishment of infection by the pathogenic yeasts. Villar et $\mathrm{al}^{45}$ have also shown that the transformation of $C$. albicans into hyphal form increases its interaction with the tissue cells, thus increasing its ability to adhere to human cells and potent invasion. 


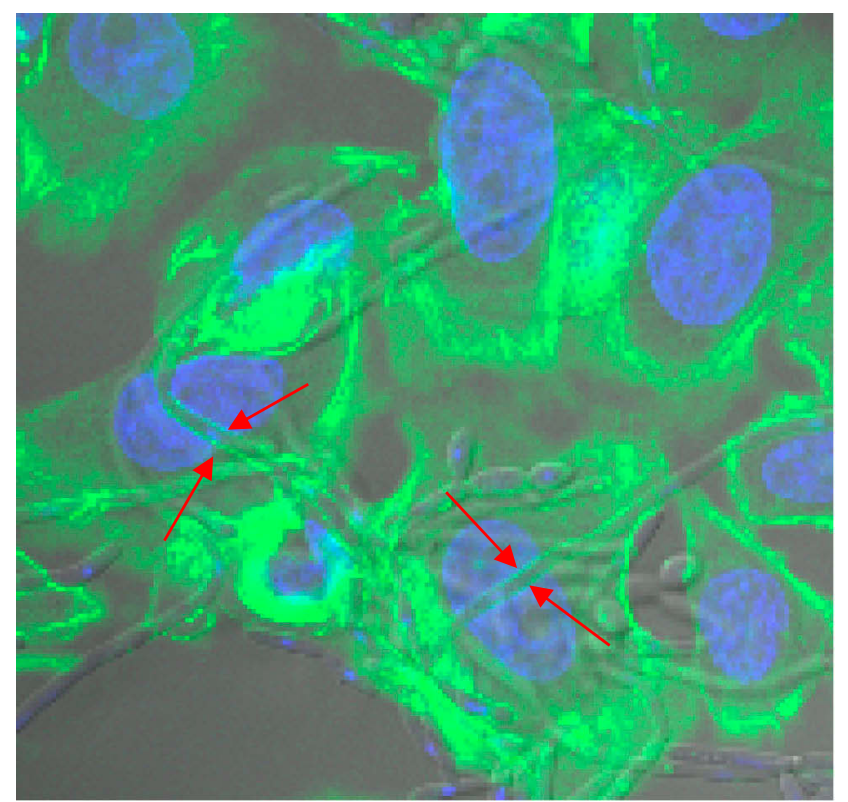

Figure 2 Confocal images of invasion of yeasts in HeLa cells. The hyphae of Candida albicans strain 8_I invaded HeLa cells. Cells from a colony of $C$. albicans 8_I were seeded with HeLa cells and incubated for 3 hours at $37^{\circ} \mathrm{C}$ and $5 \% \mathrm{CO}_{2}$. Actin remodeling was observed by staining cells with FITC-Phalloidin and red arrow indicates the cell invasion by $C$. albicans as seen by polymerized actin (green in color) surrounding the invading hyphae. Nuclear material is stained with DAPI (blue) for easy localization of cells.

\section{Diversity of Clinical Yeast Isolates}

During the present study 176 yeasts were isolated and sequencing was done using $28 \mathrm{~S}$ rRNA gene. Identification showed that Candida was the major genus and $C$. albicans was the dominant species (43\%). Other major species of Candida were by $C$. tropicalis (28\%), C. glabrata (13\%) and C. parapsilosis (8\%). Similar observation of species distribution was reported by Kullberg and Arendrup ${ }^{46}$ and Basu et $\mathrm{al}^{15}$ in which although $C$. albicans was the dominating pathogen, it accounted for only half the isolates. C. albicans was the most dominant species in all kinds of samples except blood. In blood, C. parapsilosis was the dominant yeast followed by C. glabrata and C. tropicalis (Table 2). C. tropicalis was the second most frequent species in sputum, urine, HVS and the "other" group of samples (which included ETT, bronchial, pus, etc.). There were several uncommon yeasts ie, Cyberlindnera fabianii, Issatchenkia orientalis $(=C$. krusei $=$ P. kudriavzevii), Kluyveromyces marxianus, Kodamaea ohmeri and Clavispora lusitaniae (=Candida lusitaniae $=$ Candida obtus $=$ Saccharomyces carmosousae) were isolated during the study (Table 2, Figure 3). Candida albicans, C. tropicalis and C. glabrata were found in all four samples ie, urine, sputum, blood and HVS (Figure 4). We isolated one strain of $C$. africana, which is considered as an important causal agent of vaginal infections. ${ }^{47}$

Phylogenetic analysis using sequences from 28S rRNA gene of all 176 isolates showed a total of eleven distinct ascomycetous lineages (Figure 5). BLASTn results and phylogenetic tree construction revealed that there were eight isolates from different genera including Candida, Kluyveromyces, Kodamaea, Clavispora with less sequence similarity with previously described fungal species. These species need further characterization as potential opportunistic pathogens and are recently emerging according to the sequence-based species delineation of fungal taxonomy. Although the DNA sequencing of $28 \mathrm{~S}$ rRNA gene

Table 2 Epidemiological Data and Prevalence of Yeast Species in Different Clinical Samples

\begin{tabular}{|c|c|c|c|c|c|c|}
\hline \multirow[t]{2}{*}{ Species } & \multicolumn{6}{|c|}{ Number of Isolates from Clinical Source } \\
\hline & Blood & Sputum & Urine & HVS & Others $^{\mathrm{a}}$ & Total \\
\hline Candida africana & 0 & 0 & I & 0 & 0 & I \\
\hline Candida albicans & I & 16 & 30 & 13 & 15 & 75 \\
\hline Candida glabrata & 3 & 3 & 8 & 4 & 4 & 22 \\
\hline Candida parapsilosis & 4 & 2 & 6 & 1 & 1 & 14 \\
\hline Candida tropicalis & 3 & 7 & 26 & 7 & 7 & 50 \\
\hline Clavispora lusitaniae & 0 & 3 & 1 & 0 & 0 & 4 \\
\hline Cyberlindnera fabianii & 2 & 0 & 0 & 0 & 0 & 2 \\
\hline Issatchenkia orientalis & 0 & 0 & I & 0 & I & 2 \\
\hline Kluyveromyces marxianus & 0 & I & 2 & 0 & 0 & 3 \\
\hline Kodamaea ohmeri & I & 0 & 0 & 0 & 0 & 1 \\
\hline Lodderomyces sp. & 0 & 1 & 0 & 0 & 0 & 1 \\
\hline Trichosporon asahii & I & 0 & 0 & 0 & 0 & 1 \\
\hline
\end{tabular}

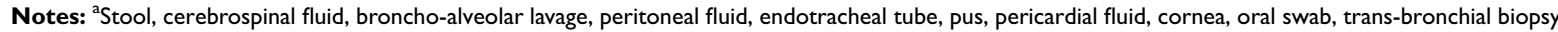




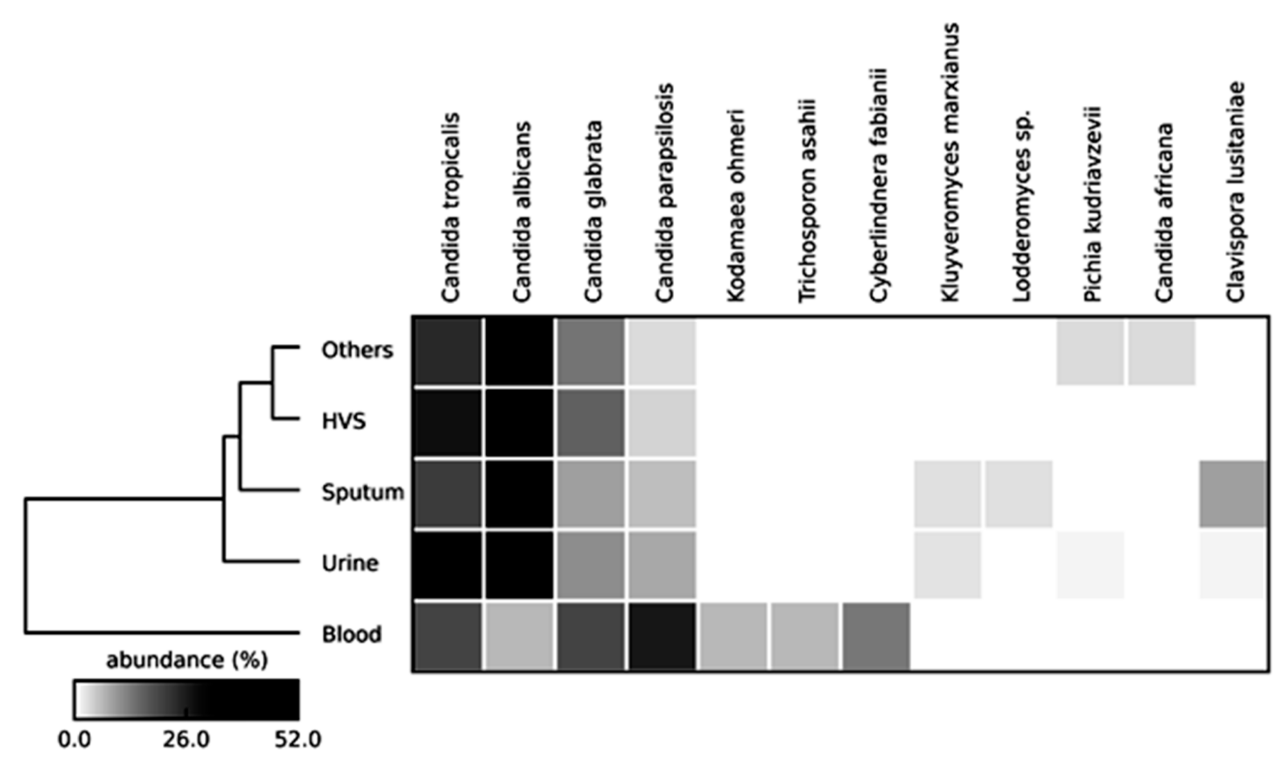

Figure 3 Venn diagram exhibiting the yeast species shared and unique to the different clinical samples.

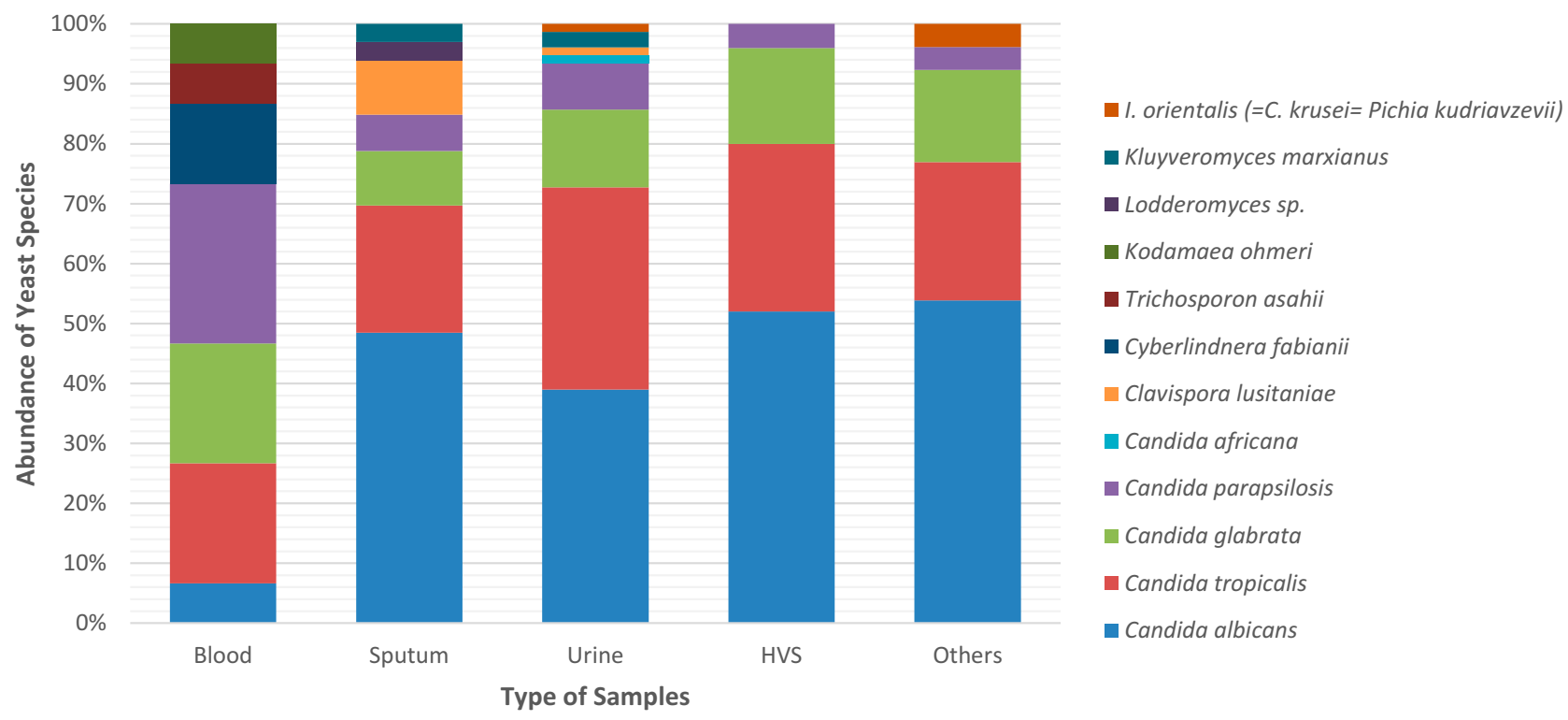

Figure 4 Relative abundance of yeasts species isolated from different clinical sources.

did not resolve C. africana and C. albicans, the identity of isolate 4_2 was retained as C. africana based on the $99 \%$ similarity with the type strain of C. africana (AY342214). Borman et $\mathrm{al}^{48}$ has shown that $C$. africana can be distinguished from $C$. albicans by pyrosequencing of a short region of ITS2. DNA sequence databases of yeast are voluminous but not completely curated. Therefore, simple sequence analysis is not sufficient for correct identification but may require in some cases the phylogenetic analysis. ${ }^{49}$

\section{Conclusion}

MALDI-TOF MS is able to identify common species but its effectiveness is restricted when it comes across uncommon species of Candida or members of uncommon genera. A regular update of the MALDI-TOF MS database is required with the addition of chromatogram of ribosomal proteins of new pathogenic strains. In our study, the accuracy of MALDITOF MS technique was nearly $90 \%$ for general yeast samples and $94 \%$ for $C$. albicans. This study reports using characteristic 


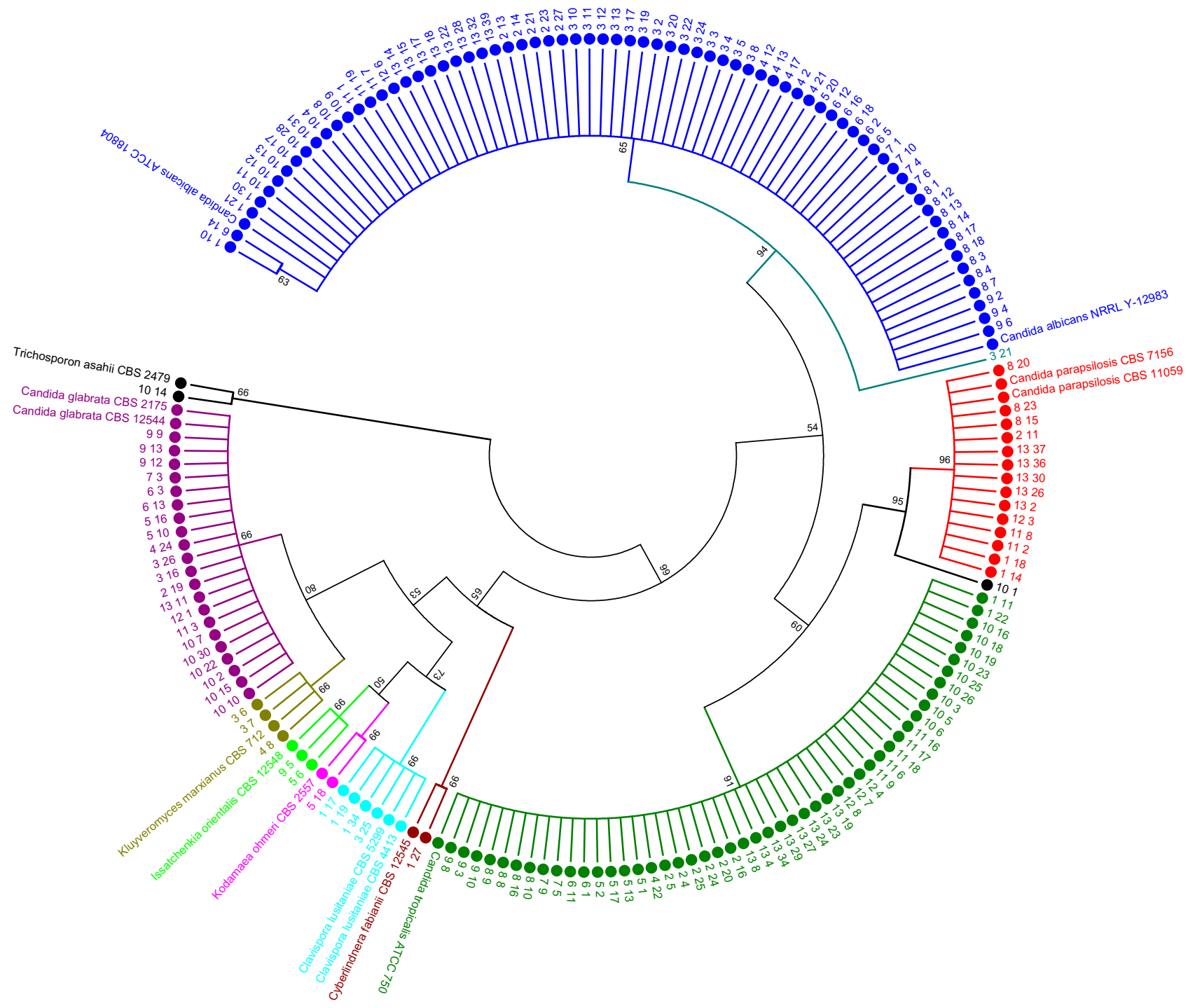

Figure 5 Phylogenetic tree constructed using 28S rRNA gene sequences of 176 isolated yeasts strains along with homologous sequences from type strains of closest match in GenBank. The evolutionary history was inferred using the $\mathrm{NJ}$ method. The optimal tree with the sum of branch length $=1.29$ shown. The evolutionary distances were computed using the Kimura 2-parameter method and are in the units of the number of base substitutions per site. The analysis involved 190 nucleotide sequences. There were a total of 694 positions in the final dataset. Evolutionary analyses were conducted in MEGA7.

MALDI-TOF MS spectra to separate successfully identified non-candida yeasts like $C y$. fabianii, K. marxianus, Ko. ohmeri and T. asahii. In recent times, the popularity and use of MALDI-TOF MS has increased which is represented in the number of publications and improvement in the technology. It is an important tool in clinical set up for microbial biotyping as it is fast, low-cost, simple to use and has wide spectrum applications in the identification of bacteria, archaea, and fungi. ${ }^{29}$ Earlier, DNA sequencing was considered better because of the rich sequence database and accurate yeast identification. A comparative analysis of these tests has been reported earlier which indicated in general conformity between the results obtained by different techniques. ${ }^{50,51}$ Phenotypic methods have been widely used by pathological laboratories due to their low cost. However, their sensitivity and reproducibility are the two disadvantages of the technique as the former depends on the morphology and physiology of the pathogen and the latter depends on the conditions of growth (which usually varies between laboratory). Thus, the chromogenic agar test although simple to perform, does not help in reliable identification as observed in this study. In addition, the time required to obtain chromogen is generally more than 2 days.

However, there is an urgent need to identify the species directly from the sample (without culturing the causal organism), if the causal organism is the only one and there is no mixed culture involved. Recently, Clancy and 


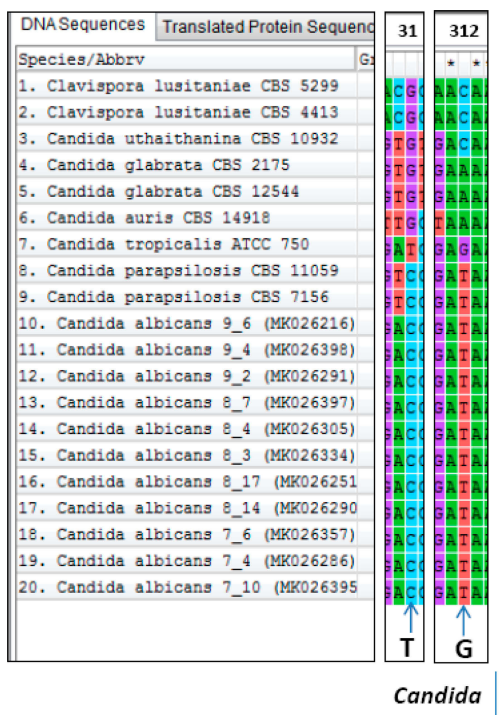

tropicalis
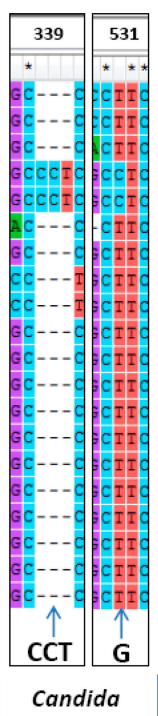

glabrata
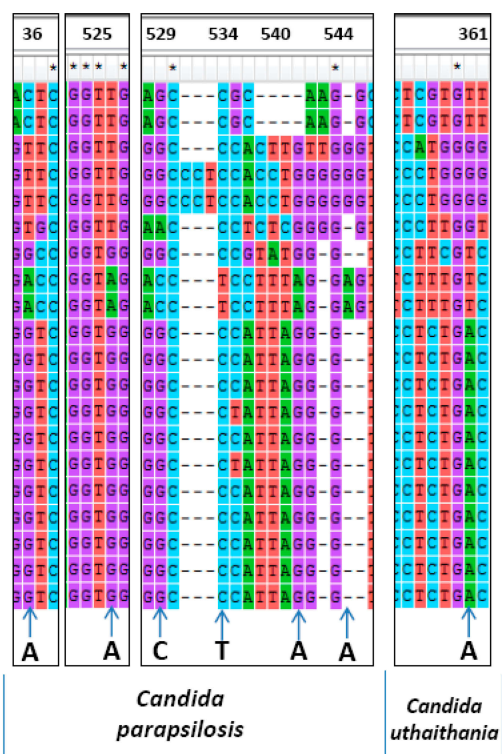

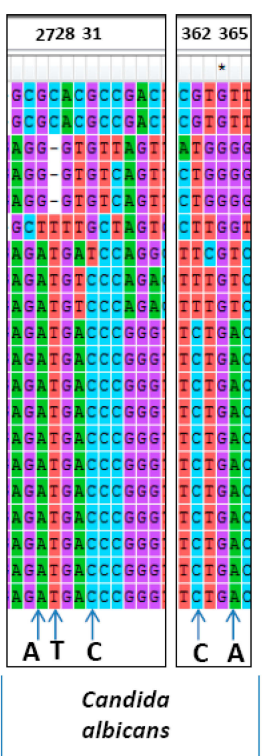

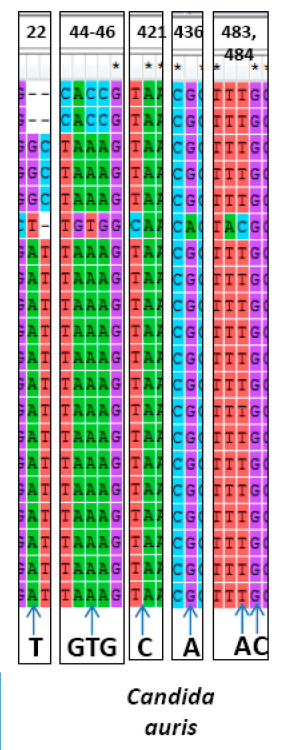

Figure 6 Nucleotide changes at various loci in the LSU region of rRNA gene among various species of Candida which can be considered as species specific markers. A total of 20 sequences (including nine sequences from the database) consisting of 535 sites has been aligned in CLUSTALW using MEGA7.0 software.

Nguyen $^{52}$ also emphasized the urgency for development and validation of non-culture diagnostic tests. It will greatly save on time of treatment which is crucial in serious cases like invasive candidiasis. At present, both the methods which are considered reliable require pure culture, ie, DNA sequencing and MALDI-TOF MS. Although MALDI based identifications are very rapid, correct identification by MALDI-TOF MS is dependent on experimental variables such as amount of biological sample, its growth phase, method employed for the sample preparation and the quality of the raw spectra obtained during general identification runs. Most of the time it is essential to obtain a high-quality real-time mass spectra which results in increased score values leading to correct identifications. We evaluated three methods of clinical yeast identification, DNA sequencing, MALDI-TOF MS and chromogenic media and, as per the observation in this study, sequencing is the best method of identification followed by MALDI-TOF MS method. As observed in this study many isolates of common yeasts were misidentified by chromogenic agar. Such kinds of misidentification impacts the clinical management of fungal disease and hence phenotypic identification is not reliable in the identification of closely related species.

Development of antifungal resistance is increasing worldwide amongst the clinical isolates of different yeasts and the present study also observed a similar phenomenon. A large number of isolates showed resistance to all the antifungals tested and also had an invasive character which is a major cause of apprehension. While azoles have long been used as the most common antifungal, relatively recently there have been several studies related to reduced susceptibility against yeasts, particularly Candida. Nowadays, high level resistance of yeast strains to azoles is one of the major and critical problems in yeasts isolated from clinical set up. A molecular analysis of all these strains is essential to substantiate the cause and mechanism of resistance. In the present study, nystatin was found to be the most effective antifungal agent. The most recently reported species of Candida, namely C. auris, frequently does not respond to normally used antifungal drugs, making infections difficult to treat. The prevalence of infection by $C$. auris is increasing. Also due to its multi-drug resistance $C$. auris infections usually have higher mortality rates which are a cause of concern. ${ }^{53}$ Increased speed and accuracy of strain identification is mandatory for therapeutics of the patient. As observed by others and in our study many new types of yeast are reported as probable human pathogens which should be included in diagnostic protocols. ${ }^{54}$ In the present study, a total of six species viz., Cy. fabianii, T. asahii, C. africana, K. marxianus, Lodderomyces sp., Ko. ohmeri are saprophytic yeasts, but isolated as pathogens from patients (Table S4). In our recent studies, many saprophytic fungi are now being reported as human pathogens e.g., Achaetomium strumarium and 
Colletotrichum gloeosporioides. ${ }^{55,56}$ Thus this study is important as it examined a large number of Indian clinical samples for yeast infection reporting opportunistic and emerging pathogens.

There are very few studies of yeast infections in an Indian context. In the present study, we isolated 176 yeast isolates from various clinical samples and evaluated their genetic diversity based on DNA sequence analysis of $28 \mathrm{~S}$ rRNA gene region. The DNA sequence analysis of different yeasts indicated that extensive genetic diversity exists even amongst each species. Brandt and Lockhart, ${ }^{57}$ reviewed important pathogenic yeast genera like Candida, Cryptococcus, Rhodotorula, Trichosporon and Malassezia for the clinical impacts of taxonomic changes. DNA sequence databases of yeast are voluminous but not completely curated and therefore simple sequence analysis is not sufficient for correct identification but may require in some cases the phylogenetic tree analysis as seen in this study. Genus specific nucleotide changes have been used by Sharma and Shouche ${ }^{58}$ for the dermatophytic fungi. Similarly, species specific nucleotides changes in the LSU region of rRNA gene among various species of Candida can also be a differentiation factor (Figure 6).

\section{Abbreviations}

ITS, internal transcribed spacer; LSU, large subunit; D1/D2, domain one and two; DAPI, 4',6-diamidino-2-phenylindole; DNA, deoxyribose nucleic acid; MALDI-TOF MS, matrix assisted laser desorption/ionization- time of flight mass spectroscopy; FITC, fluorescein isothiocyanate; FASTA, FASTAll; BLASTn, basic local alignment search tool nucleotide; NCBI, National Center for Biotechnology Information; MEGA, molecular evolutionary genetics analysis; ABI, Applied Biosystems; PCR, polymerase chain reaction; EDTA, ethylenediaminetetraacetic acid; SDA, Sabouraud Dextrose Agar; CSF, cerebrospinal fluid; ETT, endotracheal tube; PEG-NaCl, polyethylene glycol-sodium chloride; HVS, high vaginal swab.

\section{Ethics and Consent Statement}

The authors declare that the clinical samples were part of routine hospital procedure.

\section{Acknowledgments}

The authors thank the Department of Biotechnology, Government of India, New Delhi for financial support for establishing the National Centre for Microbial Resource (NCMR) project no. BT/Coord.II/01/03/2016.

\section{Funding}

No external funding was used for the work.

\section{Disclosure}

The authors report no conflicts of Interest in this work.

\section{References}

1. Pappas PG, Lionakis MS, Arendrup MC, et al. Invasive candidiasis. Nat Rev Dis Primers. 2018;4:18026. doi:10.1038/nrdp.2018.26

2. Falagas ME, Roussos N, Vardakas KZ. Relative frequency of albicans and the various non-albicans Candida spp. among Candidemia isolates from inpatients in various parts of the world: a systematic review. Int J Infect Dis. 2010;14:e954-e966. doi:10.1016/j.ijid.2010.04.006

3. Schwartz IS, Patterson TF. The emerging threat of antifungal resistance in transplant infectious diseases. Curr Infect Dis Rep. 2018;20 (3):2. doi:10.1007/s11908-018-0608-y

4. Paolucci M, Landini MP, Sambri V. How can the microbiologist help in diagnosing neonatal sepsis? Int J Pediatr. 2012;120139.

5. He S, Hang JP, Zhang L, et al. A systematic review and meta-analysis of diagnostic accuracy of serum 1, 3- $\beta$-D-glucan for invasive fungal infection: focus on cut off levels. J Microbiol Immunol Infect. 2015;48:351-361. doi:10.1016/j.jmii.2014.06.009

6. Williams DW, Lewis MAO. Isolation and identification of Candida from the oral cavity. ISRN Dent. 2000;3-11.

7. Zhang J, Hung GC, Nagamine K, et al. Development of Candida-specific real-time PCR assays for the detection and identification of eight medically important Candida species. Microbiol Insights. 2016;9:21-28. doi:10.4137/MBI.S38517

8. Avni T, Leibovici L, Paul M. PCR diagnosis of invasive Candidiasis: systematic review and meta-analysis. J Clin Microbiol. 2011; 49:665-670. doi:10.1128/JCM.01602-10

9. Brinkman NE, Haugland RA, Wymer LJ, et al. Evaluation of a rapid, quantitative real-time PCR method for enumeration of pathogenic Candida cells in water. Appl Environ Microbiol. 2003;69:1775-1782. doi:10.1128/AEM.69.3.1775-1782.2003

10. Mirhendi H, Makimura K, Khoramizadeh M, et al. A one-enzyme PCR-RFLP assay for identification of six medically important Candida species. Nihon Ishinkin Gakkai Zasshi. 2006;47:225-229. doi:10.3314/jjmm.47.225

11. Raja HA, Miller AN, Pearce CJ, et al. Fungal identification using molecular tools: a primer for the natural products research community. J Nat Prod. 2017;80:756-770. doi:10.1021/acs.jnatprod.6b01085

12. Lacroix C, Gicquel A, Sendid B, et al. Evaluation of two matrix-assisted laser desorption ionization-time of flight mass spectrometry (MALDI-TOF MS) systems for the identification of Candida species. Clin Microbiol Infect. 2014;20:153-158. doi:10.1111/1469-0691.12210

13. Qian J, Cutler JE, Cole RB, et al. MALDI-TOF mass signatures for differentiation of yeast species, strain grouping and monitoring of morphogenesis markers. Anal Bioanal Chem. 2008;392:439-449. doi:10.1007/s00216-008-2288-1

14. Pfaller MA, Houston A, Coffmann S. Application of CHROM agar candida for rapid screening of clinical specimens for Candida albicans, Candida tropicalis, Candida krusei, and Candida (Torulopsis) glabrata. J Clin Microbiol. 1996;34:58-61. doi:10.1128/JCM.34.1.58-61.1996

15. Basu S, Gugnani HC, Joshi S, et al. Distribution of Candida species in different clinical sources in Delhi, India, and proteinase and phospholipase activity of Candida albicansisolates. Rev Iberoam Micol. 2003;20:137-140. 
16. Mohandas V, Ballal M. Distribution of Candida species in different clinical samples and their virulence: biofilm formation, proteinase and phospholipase production: a study on hospitalized patients in Southern India. J Glob Infect Dis. 2011;3:4. doi:10.4103/0974-777X.77288

17. Kaur R, Dhakad MS, Goyal R, et al. Emergence of non-albicans Candida species and antifungal resistance in intensive care unit patients. Asian Pac J Trop Biomed. 2016;6:455-460. doi:10.1016/j. apjtb.2015.12.019

18. Bhattacharjee P. Epidemiology and antifungal susceptibility of Candida species in a tertiary care hospital, Kolkata, India. Curr. Med. Mycol. 2016;2. doi:10.18869/acadpub.cmm.2.2.5

19. Boratyn GM, Camacho C, Cooper PS, et al. BLAST: a more efficient report with usability improvements. Nucleic Acids Res. 2013;41: W29-33. doi:10.1093/nar/gkt282

20. Larone DH. Medically Important Fungi: A Guide to Identification. 4th ed. Washington D.C: American Society for Microbiology Press; 2002:409.

21. Kumar S, Stecher G, Tamura K. MEGA7: molecular Evolutionary Genetics Analysis Version 7.0 for bigger datasets. Mol Biol Evol. 2016;33:1870-1874. doi:10.1093/molbev/msw054

22. Chao QT, Lee TF, Teng SH, et al. Comparison of the accuracy of two conventional phenotypic methods and two MALDI-TOF MS systems with that of DNA sequencing analysis for correctly identifying clinically encountered yeasts. PLoS One. 2014;9:e109376. doi:10.1371/journal. pone. 0109376

23. Stevenson LG, Drake SK, Shea YR, et al. Evaluation of matrix-assisted laser desorption ionization - Time of flight mass spectrometry for identification of clinically important yeast species. J Clin Microbiol. 2010;48:3482-3486. doi:10.1128/JCM.00687-09

24. Mancini N, De Carolis E, Infurnari L, et al. Comparative evaluation of the Bruker Biotyper and Vitek MS matrix-assisted laser desorption ionization-time of flight (MALDI-TOF) mass Spectrometry systems for identification of yeasts of medical importance. J Clin Microbiol. 2013;51:2453-2457. doi:10.1128/JCM.00841-13

25. Sendid B, Ducoroy P, François N, et al. Evaluation of MALDI-TOF mass spectrometry for the identification of medically-important yeasts in the clinical laboratories of Dijon and Lille hospitals. Med Mycol. 2013;51:25-32. doi:10.3109/ 13693786.2012.693631

26. Westblade LF, Jennemann R, Branda JA, et al. Multicenter study evaluating the Vitek MS system for identification of medically important yeasts. J Clin Microbiol. 2013;51:2267-2272. doi:10.1128/JCM.00680-13

27. Hamprecht A, Christ S, Oestreicher T, et al. Performance of two MALDI-TOF MS systems for the identification of yeasts isolated from bloodstream infections and cerebrospinal fluids using a time-saving direct transfer protocol. Med Microbiol Immunol. 2014;203:93-99. doi:10.1007/s00430-013-0319-9

28. Li MC, Chang TC, Chen HM, et al. Oligonucleotide array and VITEK matrix-assisted laser desorption ionization-time of flight mass spectrometry in species identification of blood yeast isolates. Front Microbiol. 2018;9:1-7. doi:10.3389/fmicb.2018.00001

29. Rahi P, Prakash O, Shouche YS. Matrix-assisted laser desorption/ionization time-of-flight mass-spectrometry (MALDI-TOF MS) based microbial identifications: challenges and scopes for microbial ecologists. Front Microbiol. 2016;7:1359. doi:10.3389/fmicb.2016.01359

30. Liu H, Du Z, Wang J, Yang R. Universal sample preparation method for characterization of bacteria by matrix-assisted laser desorption ionization-time of flight mass spectrometry. Appl Environ Microbiol. 2007;73:1899-1907. doi:10.1128/AEM.02391-06

31. Freiwald A, Sauer S. Phylogenetic classification and identification of bacteria by mass spectrometry. Nat Protoc. 2009;4(5):732-742. doi: $10.1038 /$ nprot.2009.37

32. Faria-Ramos I, Neves-Maia J, Ricardo E, et al. Species distribution and in vitro antifungal susceptibility profiles of yeast isolates from invasive infections during a Portuguese multicenter survey. Eur J Clin Microbiol Infect Dis. 2014;33(12):2241-2247. doi:10.1007/ s10096-014-2194-8
33. Guinea J, Zaragoza Ó, Escribano P, et al. Molecular identification and antifungal susceptibility of yeast isolates causing fungemia collected in a population-based study in Spain in 2010 and 2011. Antimicrob Agents Chemother. 2014;58(3):1529-1537. doi:10.1128/AAC.02155-13

34. Minea B, Nastasa V, Moraru RF, et al. Species distribution and susceptibility profile to fluconazole, voriconazole and MXP-4509 of 551 clinical yeast isolates from a Romanian multi-centre study. Eur J Clin Microbiol Infect Dis. 2014;34:367-383. doi:10.1007/s10096-014-2240-6

35. Orasch C, Marchetti O, Garbino J, et al. Candida species distribution and antifungal susceptibility testing according to European committee on antimicrobial susceptibility Testing and new vs. old clinical and laboratory standards institute clinical breakpoints: a 6-year prospective candidaemia's. Clin Microbiol Infect. 2014;20:698-705. doi:10.1111/1469-0691.12440

36. Meletiadis J, Curfs-Breuker I, Meis JF, et al. In vitro antifungal susceptibility testing of Candida isolates with the EUCAST methodology, a new method for ECOFF determination. Antimicrob Agents Chemother. 2017;61:1-6. doi:10.1128/AAC.02372-16

37. Bernhardt J, Bernhardt $H$, Knoke M, et al. Influence of voriconazole and fluconazole on reconstituted multilayered oesophageal epithelium infected by Candida albicans. Mycoses. 2004;47:330-337. doi:10.11 11/j.1439-0507.2004.01004.x

38. Wächtler B, Wilson D, Hube B. Candida albicans adhesion, invasion and damage of vaginal epithelial cells: stage-specific inhibition by clotrimazole and bifonazole. Antimicrob Agents Chemother. 2011;55 (9):4436-4439. doi:10.1128/AAC.00144-11

39. Martins HP, Da Silva MC, Paiva LC, et al. Efficacy of fluconazole and nystatin in the treatment of vaginal Candida species. Acta Derm Venereol. 2012;92:78-82.

40. Whaley SG, Berkow EL, Rybak JM, Nishimoto AT, Barker KS, Rogers PD. Azole antifungal resistance in Candida albicans and emerging non-albicans Candida Species. Front Microbiol. 2017;7: 2173. doi:10.3389/fmicb.2016.02173

41. Castanheira M, Messer SA, Jones RN, Farrell DJ, Pfaller MA. Activity of echinocandins and triazoles against a contemporary (2012) worldwide collection of yeast and moulds collected from invasive infections. Int $J$ Antimicrob Agents. 2014;44:320-326. doi:10.1016/j.jjantimicag.2014.06.007

42. Pfaller MA, Castanheira M, Lockhart SR, Ahlquist AM, Messer SA, Jones RN. Frequency of decreased susceptibility and resistance to echinocandins among fluconazole-resistant bloodstream isolates of Candida glabrata. J Clin Microbiol. 2012;50:1199-1203. doi:10.1128/ JCM.06112-11

43. Cho EJ, Shin JH, Kim SH, et al. Emergence of multiple resistance profiles involving azoles, echinocandins and amphotericin B in Candida glabrata isolates from a neutropenia patient with prolonged fungaemia. $J$ Antimicrob Chemother. 2015;70:1268-1270. doi:10.1093/jac/dku518

44. Sun JN, Solis NV, Phan QT, et al. Host cell invasion and virulence mediated by Candida albicans Ssa1. PLoS Pathog. 2010;6(11): e1001181. doi:10.1371/journal.ppat.1001181

45. Villar CC, Kashleva H, Mitchell AP, et al. Invasive phenotype of Candida albicans affects the host pro-inflammatory response to infection. Infect Immun. 2005;73(8):4588-4595. doi:10.1128/ IAI.73.8.4588-4595.2005

46. Kullberg BJ, Arendrup MC. Invasive Candidiasis. $N$ Engl $J$ Med. 2016;374:794-795. doi:10.1056/NEJMc1514201

47. Romeo O, Tietz HJ, Criseo G. Candida africana: is it a fungal pathogen? Curr Fungal Infect Rep. 2013;7:192-197. doi:10.1007/s12281-013-0142-1

48. Borman AM, Szekely A, Linton CJ, et al. Epidemiology, antifungal susceptibility, and pathogenicity of Candida africana isolates from the United Kingdom. J Clin Microbiol. 2013;51:967-972. doi:10.1128/JCM.02816-12

49. Yokoyama K, Biswas SK, Miyaji M, Nishimura K. Identification and phylogenetic relationship of the most common pathogenic Candida species inferred from mitochondrial cytochrome $\mathrm{b}$ gene sequences. J Clin Microbiol. 2000;38:4503-4510. doi:10.1128/JCM.38.12.45034510.2000 
50. Aubertine CL, Rivera M, Rohan SM, et al. Comparative study of the new colorimetric VITEK 2 yeast identification card versus the older fluorometric card and of CHROM agar Candida as a source medium with the new card. J Clin Microbiol. 2006;44:227-228. doi:10.1128/ JCM.44.1.227-228.2006

51. Wang H, Fan YY, Kudinha T, et al. A comprehensive evaluation of the Bruker Biotyper MS and Vitek MS Matrix-Assisted Laser Desorption Ionization-Time of Flight Mass spectrometry systems for identification of yeasts, part of the national China hospital invasive fungal surveillance net (CHIF-NET). $J$ Clin Microbiol. 2016;54:1376-1380. doi:10.1128/JCM.00162-16

52. Clancy CJ, Nguyen MH. Diagnosing invasive candidiasis. $J$ Clin Microbiol. 2018;56:e1909-17. doi:10.1128/JCM.01909-17

53. Chowdhary A, Sharma C, Meis JF. Candida auris: a rapidly emerging cause of hospital-acquired multidrug-resistant fungal infections globally. PLoS Pathog. 2017;13(5):e1006290. doi:10.1371/journal. ppat. 1006290
54. Eddouzi J, Hofstetter V, Groenewald M. Characterization of a new clinical yeast species, Candida tunisiensis sp. nov., isolated from a strain collection of Tunisian hospitals. J Clin Microbiol. 2012;17: JCM-01627.

55. Pote ST, Chakraborty A, Lahiri KK, et al. Keratitis by a rare pathogen Colletotrichum gloeosporioides: a case report. J. Mycol Med. 2017;27:407-411. doi:10.1016/j.mycmed.2017.04.009

56. Pote ST, Khan U, Lahiri KK, et al. Onychomycosis due to Achaetomium strumarium. J. Mycol Med. 2018;28:510-513. doi:10.1016/j.mycmed.2018.07.002

57. Brandt ME, Lockhart SR. Recent taxonomic developments with Candida and other opportunistic yeasts. Curr Fungal Infect Rep. 2012;6(3):170-177. doi:10.1007/s12281-012-0094-x

58. Sharma R, Shouche Y. Nannizzia graeserae sp. nov., a new dermatophyte of geophilic clade isolated from vicinity of a barbershop in India. Kavaka. 2018;50:14-20.
Infection and Drug Resistance

\section{Publish your work in this journal}

Infection and Drug Resistance is an international, peer-reviewed openaccess journal that focuses on the optimal treatment of infection (bacterial, fungal and viral) and the development and institution of preventive strategies to minimize the development and spread of resistance. The journal is specifically concerned with the epidemiology of
Dovepress

antibiotic resistance and the mechanisms of resistance development and diffusion in both hospitals and the community. The manuscript management system is completely online and includes a very quick and fair peerreview system, which is all easy to use. Visit http://www.dovepress.com/ testimonials.php to read real quotes from published authors. 